\title{
Does the order of mutational acquisition in myeloproliferative neoplasms matter? Evidence from JAK2 exon 12 and DNMT3A co-mutant polycythemia vera
}

\author{
Rumyana Todorova ${ }^{1,2} \cdot$ Jakob Passweg $^{3} \cdot$ Pontus Lundberg $^{3} \cdot$ Alexandar Tzankov $^{1}$ \\ Received: 4 February 2020 / Accepted: 25 February 2020 / Published online: 5 March 2020 \\ (C) Springer-Verlag GmbH Germany, part of Springer Nature 2020
}

Myeloproliferative neoplasms (MPN) have in common an abnormal clonal expansion of differentiated hematopoietic cells in the bone marrow (BM), usually accompanied by excessive production of progenies leading to numeric blood cell increase. Three classical MPN types are recognizedpolycythemia vera (PV), essential thrombocythemia (ET), and primary myelofibrosis (PMF).

Characteristic somatic mutations of JAK2, MPL, and $C A L R$, considered "drivers" directly linked to the MPN phenotype, were discovered in MPN [1]. The somatic JAK2V617F gain-of-function mutation is found in $>95 \%$ of PV patients [1], while most of the small proportion of JAK2V617F-negative PV individuals bear JAK2 exon 12 mutations [2]. Both JAK2 mutations lead to constitutive activation of the JAK-STAT signaling pathway supporting the neoplastic outgrowth of the respective clone [3]. Other accompanying nondriver mutations such ASXL1, EZH2, and/or TET2 are implicated too, especially in PMF, and are able to considerably affect individual clinical course [1, 4]. These latter mutations have major impact on epigenetic modulation, enhance myeloid tumorigenesis in general, and are encountered in a variety of hematological neoplasms. Another recurrently mutated

At the time of diagnosis, Rumyana Todorova was a visiting scientist at the Institute of Mediacal Genetics and Pathology of the University Hospital of Basel.

Alexandar Tzankov

alexandar.tzankov@usb.ch

1 Institute of Medical Genetics and Pathology, University Hospital of Basel, Basel, Switzerland

2 Institute of Pathology, Ludwig Maximilian University of Munich, Munich, Germany

3 Department of Hematology, University Hospital of Basel, Basel, Switzerland gene, also encoding for an epigenetic modifier, is DNMT3A, and its loss-of-function mutations are supposed to abrogate hematopoietic cell differentiation and increase self-renewal, thus promoting tumor growth. The incidence of DNMT3A mutations in MPN is 3\% (ET) to $15 \%$ (PMF) $[5,6]$.

In December 2016 in a peripheral hospital, a 39-year-old male patient presented with visual disturbance and bilateral congestion of the orbital/periorbital veins and a slight splenomegaly of $14.5 \mathrm{~cm}$. He had peripheral blood anisopoikilocytosis and anisochromia and hemoglobin of $244 \mathrm{~g} / \mathrm{L}$, hematocrit of $75 \%$, and erythropoietin level < $1 \mathrm{U} /$ L. Empirical therapy with regular phlebotomies was started because of the risk of hyperviscosity complications, and after several courses, his values changed to $149 \mathrm{~g} / \mathrm{L}, 47 \%$, and $2.7 \mathrm{U} / \mathrm{L}$ (lower lab range $4.3 \mathrm{U} / \mathrm{L}$ ), respectively. Noticeably, the white blood cell count was in the normal range $(8.78 \mathrm{~g} / \mathrm{L})$ but with a slight neutrophilia $(6.73 \mathrm{~g} / \mathrm{L}$; upper lab range $6.7 \mathrm{~g} /$ $\mathrm{L})$, while monocytes $(0.29 \mathrm{~g} / \mathrm{L})$ and platelets $(266 \mathrm{~g} / \mathrm{L})$ were within normal ranges. Three months later, after rheological stabilization, a diagnostic BM biopsy was performed showing hypercellularity and a loose network of reticulin fibers with some intersections, especially in perivascular areas, corresponding to reticulin fibrosis grade 1 (Fig. 1a, b). All hematopoietic lineages were increased with groups of pleomorphic to small, at occasions, dysplastic megakaryocytes around sinuses forming just a few loose clusters. BM aspirate showed considerably erythroid predominance without dysplasia; some maturation disturbances in $<10 \%$ of the myelopoietic cells and pleomorphic, at occasions $(<10 \%)$, hypolobated megakaryocytes; and depleted iron stores. Conventional karyotypization yielded an unremarkable male karyotype $46, \mathrm{XY}$, as did array comparative genomic hybridization, additionally ruling out acquired uniparental disomies (aUPD). High-throughput sequencing analysis applying a customized NGS panel for "myeloid" mutations in 38 genes detected two mutations: JAK2 H538-539delinsQL (allelic burden 26\%) and 
Fig. 1 Bone marrow biopsy three months after several courses of phlebotomy showing hypercellularity (packed marrow), erythroid hyperplasia and increased amounts of atypical but loosely clustered megakaryocytes (a, HE staining), and a loose network of reticulin fibers, corresponding to reticulin fibrosis grade 1 (b, reticulin staining)

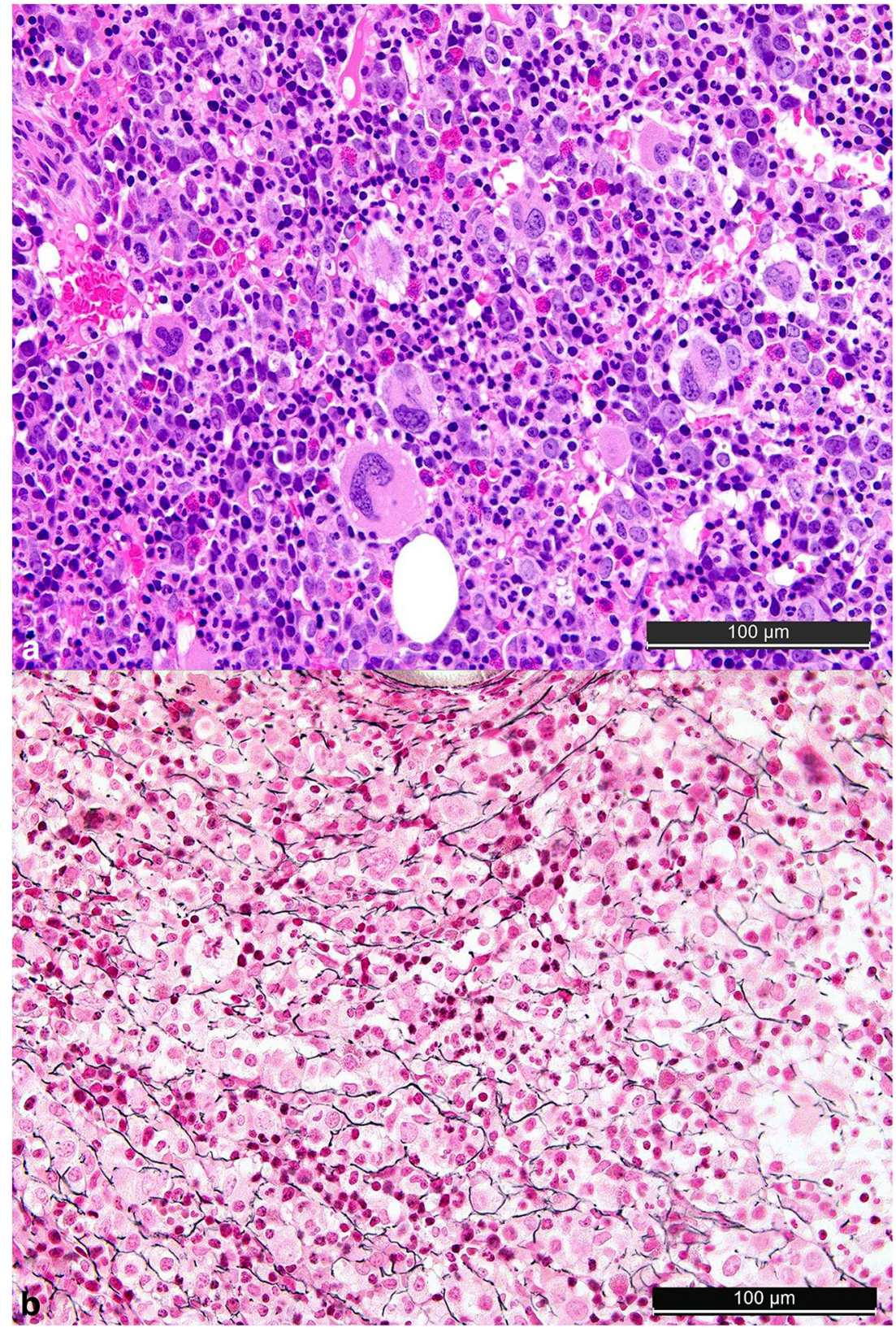

DNMT3A R771X (allelic burden 11\%). The integrative diagnosis of a chronic-phase MPN with myelofibrosis grade I and $J A K 2$ exon 12 mutation, consistent with phlebotomypretreated PV, was established. The patient was put on acetylsalicylic acid $100 \mathrm{mg}$ for six months with the need for regular phlebotomies, and then on ruxolitinib $10 \mathrm{mg}$ bid finally leading to clinical course stabilization (hematocrit $42 \%$, spleen size normalization) as per his last clinical visit in November 2019. The majority of the JAK2-V617F-negative $J A K 2$ exon 12 mutant patients are found to harbor one of the following four mutations: N542-E543del, F537-K539delinsL, K539L, and H538QK539L. Such exon 12 mutant alleles induce cytokine-independent/-hypersensitive proliferation in erythropoietin receptor-expressing cell lines by constitutive activation of the JAK-STAT signaling pathway [2, 3]. JAK2 exon 12 mutations are only observed in PV and are specific to patients with isolated erythrocytosis without concomitant leukocytosis or thrombocytosis. Several other exon 12 mutational variants have been identified [3, 7]; the mutation in our present case, i.e., H538-539delinsQL, also leads to substitution of lysine for leucine at codon 539, analogously to K539L, joining the above list.

In addition, our patient harbored a subclonal (since trisomies and aUPD for chromosomes 2 and 9 have been excluded) DNMT3A R771X mutation. Importantly, Jacquelin et al. recently provided insights into how $J A K 2-\mathrm{V} 617 \mathrm{~F}$ and DNMT3A mutations collaborate to accelerate disease progression in PV-like models by upregulating self-renewal 
programs: double-mutant cells show changes in chromatin accessibility with decreased repressive H3K27me3 marks replaced by activating $\mathrm{H} 3 \mathrm{~K} 27 \mathrm{ac}$ marks [8].

Furthermore, the order of acquisition of JAK2 and $D N M T 3 A$ mutations may influence the MPN phenotype [9]. Mostly, DNMT3A mutations precede the JAK2 mutation, which was not the case in our patient. Indeed, such order of mutational acquisition, i.e., "DNMT3A first," is rather associated with an ET phenotype. By contrast, acquisition of JAK2V617F prior to mutation of DNMT3A, i.e., "JAK2 first," is more likely associated with PV, as it was the case here. Finally, the co-occurrence of both mutations in our patient may explain the rather unusual neutrophilia, which is not common in exon 12 mutant PV, the presence of myelofibrosis, as suggested by observations of higher DNMT3A mutational frequencies in post-PV myelofibrosis [5], and possibly the occasional dysmegakaryopoiesis.

Subtle unusual details of the clinical history and the histopathology of our "JAK2 first" exon 12 and DNMT3A comutant patient, such as his rather early age of presentation, initial affection by myelofibrosis, some dysmegakaryopoiesis, packed marrow, and neutrophilia, fit with and can be likely explained by the type and sequence of mutation acquisition [9]. We think that — along with analogous observations in other cases-we contribute evidence justifying prospective clinical studies with a closer look at the mutational order in MPN to address whether this information might contribute to diagnostic and therapeutic precision.

Author contributions RT wrote the manuscript and participated in establishing the diagnosis. JP held the clinical contact and treated the patient. PL performed molecular analysis. AT partially wrote the manuscript, established the diagnosis, and took microphotographs. All authors have read, edited, and approved the manuscript.

\section{Compliance with ethical standards}

Statement of ethics The study has been conducted in accordance with the World Medical Association Declaration of Helsinki. The patient has given a written consent to publish his case.
Conflict of interest The authors declare that they have no conflicts of interest.

\section{References}

1. Vainchenker W, Kralovics R (2017) Genetic basis and molecular pathophysiology of classical myeloproliferative neoplasms. Blood 129:667-679

2. Scott LM, Thong W, Levine RL et al (2007) JAK2 exon 12 mutations in polycythemia vera and idiopathic erythrocytosis. $\mathrm{N}$ Engl $\mathrm{J}$ Med 356:459-468

3. Butcher CM, Hahn U, To LB et al (2008) Two novel JAK2 exon 12 mutations in JAK2V617F-negative polycythaemia vera patients. Leukemia 22:870-873

4. Barbui T, Thiele J, Gisslinger H et al (2018) The 2016 WHO classification and diagnostic criteria for myeloproliferative neoplasms: document summary and in-depth discussion. Blood Cancer J 8:15

5. Stegelmann F, Bullinger L, Schlenk RF, Paschka P, Griesshammer M, Blersch C, Kuhn S, Schauer S, Döhner H, Döhner K (2011) DNMT3A mutations in myeloproliferative neoplasms. Leukemia 25:1217-1219

6. Brunetti L, Gundry MC, Goodell MA (2017) DNMT3A in leukemia. Cold Spring Harb Perspect Med 7. https://doi.org/10.1101/ cshperspect.a030320

7. Percy MJ, Scott LM, Erber WN, Harrison CN, Reilly JT, Jones FG, Green AR, McMullin M (2007) The frequency of JAK2 exon 12 mutations in idiopathic erythrocytosis patients with low serum erythropoietin levels. Haematologica 92:1607-1614

8. Jacquelin S, Straube J, Cooper L, Vu T, Song A, Bywater M, Baxter E, Heidecker M, Wackrow B, Porter A, Ling V, Green J, Austin R, Kazakoff S, Waddell N, Hesson LB, Pimanda JE, Stegelmann F, Bullinger L, Döhner K, Rampal RK, Heckl D, Hill GR, Lane SW (2018) Jak2V617F and Dnmt3a loss cooperate to induce myelofibrosis through activated enhancer-driven inflammation. Blood 132: 2707-2721

9. Nangalia J, Nice FL, Wedge DC, Godfrey AL, Grinfeld J, Thakker C, Massie CE, Baxter J, Sewell D, Silber Y, Campbell PJ, Green AR (2015) DNMT3A mutations occur early or late in patients with myeloproliferative neoplasms and mutation order influences phenotype. Haematologica 100:e438-e442

Publisher's note Springer Nature remains neutral with regard to jurisdictional claims in published maps and institutional affiliations. 